\title{
Addendum: The effect of early life experience, environment, and genetic factors on spontaneous home-cage aggression-related wounding in male C57BL/6 mice
}

\author{
Brianna N. Gaskill, Aurora M. Stottler, Joseph P. Garner, Christina W. Winnicker, Guy B. Mulder and
} Kathleen R. Pritchett-Corning

Addendum to: Lab Animal https://doi.org/10.1038/laban.1225, published online 22 March 2017.

The version of this article originally published contained an error in the calculation of the weighted PALS score. In the Excel worksheet used for scoring the images (Supplementary Data), an error was made whereby the weighted section score was calculated as 'lesion score/proportion', whereas it should have been calculated as 'lesion score $\times$ proportion'. This miscalculation occurred in 82 of the total 2,700 sections that were scored, and affected the final PALS scores. To correct this error, the authors have revised Figs. 1-6 to reflect the corrected least square mean weighted PALS and associated variances, and amended the Results section to provide the revised GLM and $P$ values. The corrections in the statistical tests did not change the significance of any of the findings. In addition, in the "Substrain" subsection under "Statistical analyses" in the Materials and Methods section, the opening text should have read as follows: "A full factorial GLM was run and included the following factors: substrain of B6 and ID method, plus their interaction. The interaction of the two factors was not significant, so it was removed from the final analysis, which tested only the two main effects." Corrected figures, Results, and Supplementary Data are included in this Addendum; the original article and associated Supplementary Information published online have not been revised.

\section{Results}

Wean age and group composition. The only significant effect found in the analysis was the age of the mice at weaning $\left(\mathrm{GLM}\right.$ : $\mathrm{F}_{2,30}=13.6$; $P<0.001$; Fig. 1). A positive linear trend was found $(P<0.05)$ where 14 -d-old pups had the lowest weighted PALS and the 28 -d-old pups had the highest scores. Stability of the weaning group did not significantly explain variation in PALS $\left(\mathrm{GLM}: \mathrm{F}_{1,30}=0.03 ; P=0.84 ;\right.$ Fig. 2$)$.

Enrichment. Of the two main treatments tested, only scent was significant (GLM: $\mathrm{F}_{1,69}=5.0 ; P=0.03$; Fig. 3). Cages treated with lavender had significantly higher PALS. The enrichments tested did not significantly alter PALS (GLM: $\mathrm{F}_{1,69}=2.5 ; P=0.07$; Fig. 4 ). Time on study unsurprisingly altered wound severity as measured by PALS (GLM: $\mathrm{F}_{1,69}=38.5 ; P<0.001$ ), as the mice in 33 out of the total 80 cages had to be euthanized owing to severe aggression prior to the end of the 2 weeks. The rack on which the cage was placed, but not the location on an individual rack, was also found to significantly affect PALS (GLM: $\mathrm{F}_{1,69}=4.9 ; P=0.03$ ), with animals on one rack having a higher PALS. The average body weight of the mice in the cage was a significant covariate (GLM: $\left.\mathrm{F}_{1,69}=8.1 ; P=0.006\right)$. A positive association was found for average body weight, where cages with heavier average body weight had higher PALS.

Substrain and identification method. The substrain of B6 tested was not found to be significant (GLM: $\mathrm{F}_{5,52}=0.36 ; P=0.87$; Fig. 5). However, the ID method used did significantly affect results $\left(\mathrm{GLM}: \mathrm{F}_{1,52}=9.19 ; P=0.004 ;\right.$ Fig. 6$)$. Mice that received a tail tattoo had significantly lower PALS than those that were ear-notched.

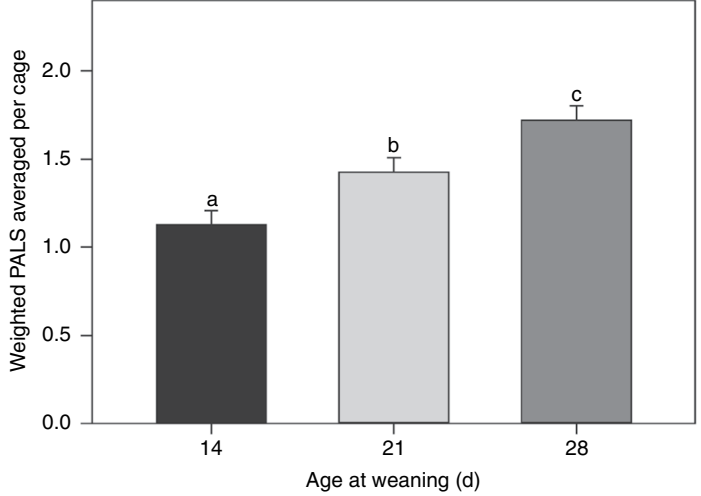

Fig. 1 | The effect of days of age at weaning on the weighted PALS. Data are presented as LSM and s.e.m. Different letters indicate a significant difference at $P<0.05 . n=12$ (cages) for each age group. See the Supplementary Data for numerical datasets.

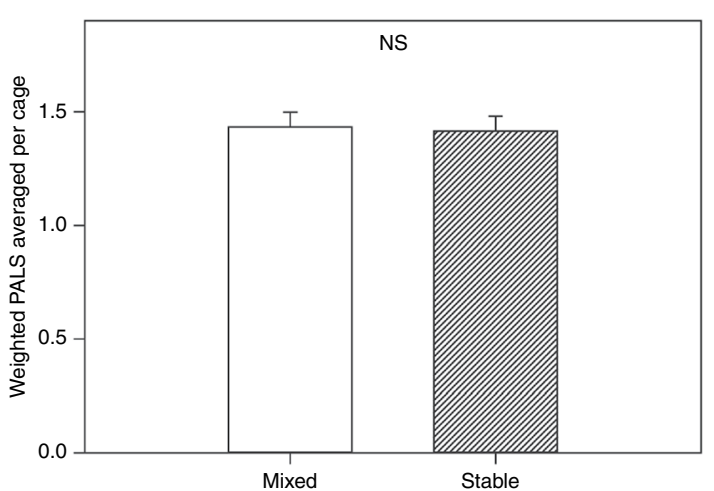

Fig. 2 | The effect of post-weaning housing of mice being either mixed weekly at cage change to combine pups of similar weight or kept in stable groups until shipment at 7 weeks of age. NS indicates no significant treatment effect found in the general linear model $(P>0.05)$. Data are presented as LSM and s.e.m. $n=18$ (cages) for mixed and stable groups. See the Supplementary Data for numerical datasets. 


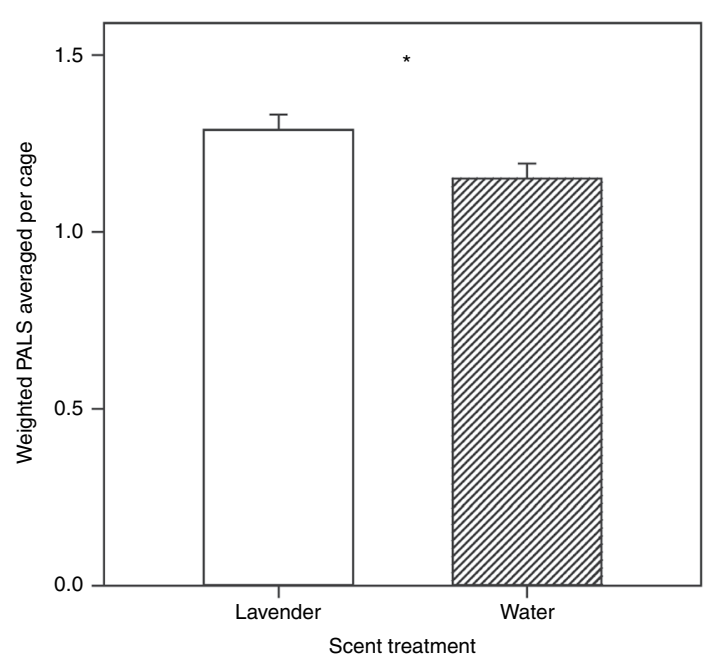

Fig. 3 | Scent treatment effects on weighted PALS. Data are presented as LSM and s.e.m. ${ }^{\star} P<0.05$, indicating a significant treatment effect found in the general linear model. $n=40$ (cages) for lavender and water groups. See the Supplementary Data for numerical datasets.

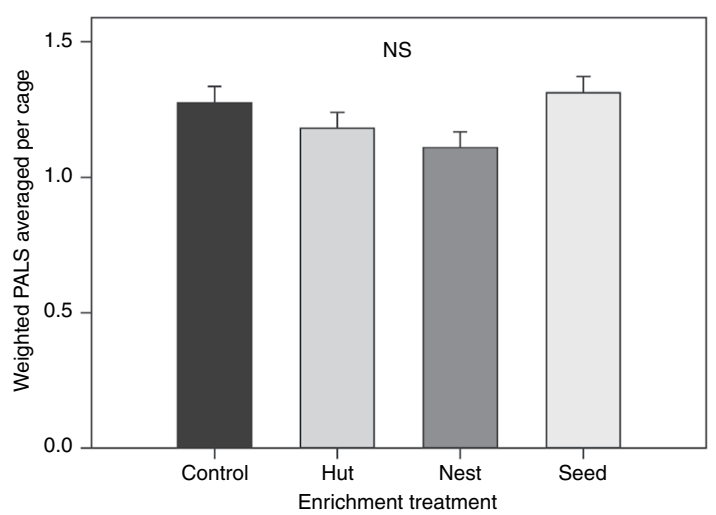

Fig. 4 | The effect of environmental treatments on PALS. NS indicates no significant treatment effect found in the general linear model $(P>0.05)$. Data are presented as LSM and s.e.m. $n=20$ (cages) for all groups. See the Supplementary Data for numerical datasets.

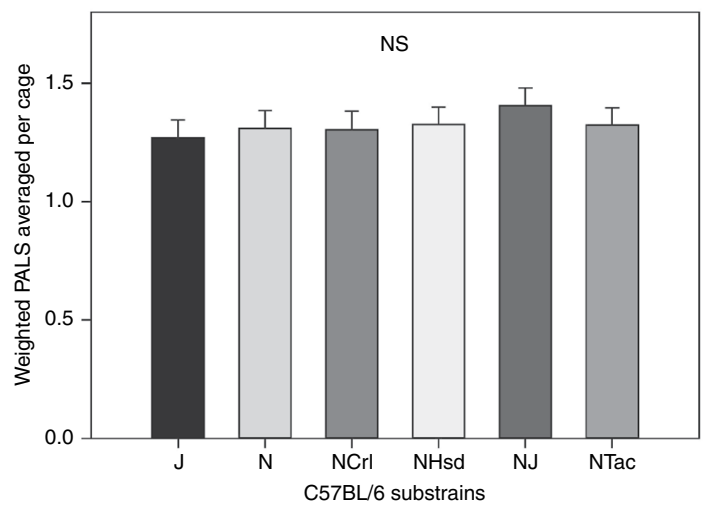

Fig. 5 | Substrain differences in end-of-study weighted PALS. NS indicates no significant treatment effect found in the general linear model $(P>0.05)$. Data are presented as LSM and s.e.m. $n$ values (cages) for the groups are as follows: $J=10, N=10, \mathrm{NCrl}=9$, $\mathrm{NHsd}=10, \mathrm{NJ}=10, \mathrm{NTac}=10$. See the Supplementary Data for numerical datasets.

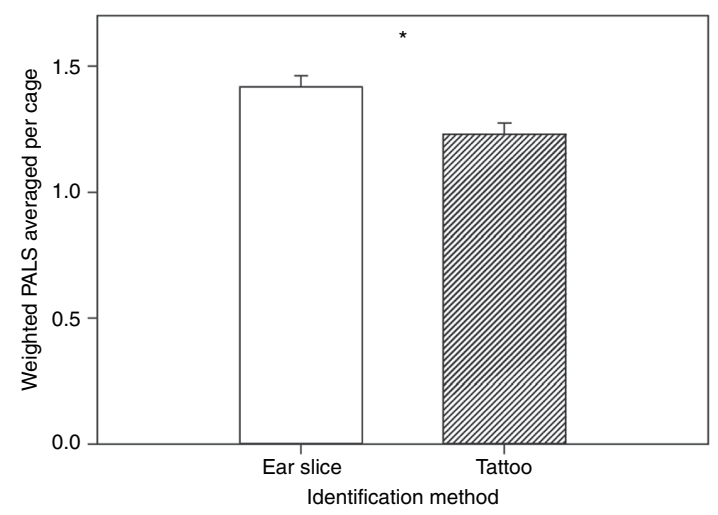

Fig. 6 | The effect of identification method on weighted PALS. Data are presented as LSM and s.e.m. ${ }^{\star} P<0.05$, indicating a significant treatment effect found in the general linear model. $n=31$ (cages) for the ear slice group; $n=29$ (cages) for the tattoo group. See the Supplementary Data for numerical datasets.

\section{Additional information}

Supplementary information is available for this paper at https://doi.org/10.1038/s41684-019-0283-0.

(c) The Author(s), under exclusive licence to Springer Nature America, Inc. 2019

Published online: 25 March 2019

https://doi.org/10.1038/s41684-019-0283-0 